\title{
A unified cosmological model for solution of problems of Cosmological constant, Cosmic coincidence, Energy conservation, Flatness and homogeneity of Horizon
}

\author{
Biswaranjan Dikshit \\ Laser and Plasma Technology Division \\ Bhabha Atomic Research Centre, Mumbai-400085, INDIA \\ And Homi Bhabha National Institute, Mumbai-400094, INDIA \\ Electronic mail: bdikshit73@yahoo.co.in
}

\begin{abstract}
Cosmological constant problem is the difference by a factor of $\sim 10^{123}$ between quantum mechanically calculated vacuum energy density and astronomically observed value. Cosmic coincidence problem questions why matter energy density is of the same order as the present vacuum energy density (former is $\sim 32 \%$ and latter is $\sim 68 \%$ ). Recently, by quantizing zero-point field of space, we have developed a cosmological model that predicts correct value of vacuum and non-vacuum energy density. In this paper, we remove some earlier assumptions and develop a generalized version of our cosmological model to solve three more problems viz. energy conservation, flatness and horizon problem along with the above two. For creation of universe without violating law of energy conservation, net energy of the universe including (negative) gravitational potential energy must be zero. However, in conventional method, its quantitative proof needs the space to be exactly flat i.e. zero-energy universe is a consequence of flatness. But, in this paper, we will prove a zero-energy universe without using flatness of space and then show that flatness is actually a consequence of zero energy density. Finally, using our model we solve the horizon problem of universe. Although cosmic inflation can explain the flatness of space and uniformity of horizon by invoking inflaton field, it cannot predict the present value of vacuum energy density or matter density. But, our cosmological model solves in an unified manner all the above mentioned five problems viz. cosmological constant problem, cosmic coincidence problem, energy conservation, flatness and horizon problem.
\end{abstract}

Keywords: Cosmological constant problem, Cosmic coincidence problem, Energy conservation, Dark energy, Flatness problem, Horizon problem 


\section{Introduction}

The human civilization has been curious to know the nature and evolution of the universe since ancient times. Long back, our observable universe with three spatial dimensions was thought to be infinite in extent and was considered to exist since infinite time. But, modern science has concluded that the universe has a finite extent and finite age. This is due to the following reason. If the universe was infinite and there is a finite density of stars, while looking at any direction, our view must hit a star. So, we will be surrounded by an environment whose temperature is same as that of stars. But, this is not the case. Similarly, if the universe existed since infinite time, all parts of it would have reached thermal equilibrium (or same temperature) by now by exchanging radiations. But, we do see different temperatures on different objects in the universe. So, we have now concluded that the universe has a finite extent and finite age. Consequent to discovery of General theory of Relativity by Einstein and observation of expansion of the universe by Hubble, Big-Bang model was proposed in which universe was assumed to be created by a big explosion of radiation and matter and its age was estimated to be $\sim 14$ billion years. However, Big-Bang model of universe suffered from five major problems viz. Cosmological constant problem, Cosmic coincidence problem, Energy conservation, Flatness and Horizon problem. At first, we will briefly explain all these problems and then proceed to develop a unified cosmological model to solve them.

\section{Cosmological constant problem and cosmic coincidence problem:}

Our universe is expanding. From the observed rate of expansion of the universe and since space is observed to be flat, solution of Einstein's field equations i.e. Friedmann's equation indicates that total energy density due to matter, radiation and vacuum must be equal to critical density $\left(\rho_{\text {crit }}=\frac{3 H^{2}}{8 \pi G}\right)$ where $H$ is Hubble's constant. On measurement, we find that matter energy including dark matter can account only $\sim 32 \%$ of this critical density. Radiation energy is found to be less than $0.1 \%$. So, it is estimated that rest $\sim 68 \%$ of the total energy of the universe must be vacuum energy (dark energy). Numerical value of this vacuum energy density comes out to be $5.3 \times 10^{-10} \mathrm{~J} / \mathrm{m}^{3}$. However, as shown by Weinberg (1989), quantum mechanically calculated vacuum energy density comes out to be $\pi^{2} \hbar c / l_{p}^{4}=10^{114} \mathrm{~J} / \mathrm{m}^{3}$ (where $l_{p}$ is Planck length) which is $\sim 10^{123}$ times more than expected astronomical value. This is known as cosmological constant problem [1-2]. 
Another significant problem in cosmology is the question, why is the matter energy density of the same order as the vacuum energy density today? This is surprising as matter energy density decreases as $1 / R^{3}$ where as vacuum energy density is assumed to be constant. This problem is known as cosmic coincidence problem [3].

\section{Problem of conservation of energy:}

Presently total matter (including dark part) in the universe is estimated to be about $\sim 10^{54} \mathrm{~kg}$. The question is, what was the source of such a huge amount of energy? A quantum vacuum fluctuation can at best create Planck energy of $\sim 0.02 \mathrm{mg}$ in a Planck time of $5.4 \times 10^{-44} \mathrm{sec}$. To answer this question, it was proposed that sum total of energy of the universe due to matter/vacuum and gravitation potential energy might be zero so that the law of conservation of energy of the universe is satisfied since its creation. This is possible as the sign of gravitational potential energy is negative whereas that of other forms is positive. To make net energy zero, magnitude of gravitational potential energy must be equal to the magnitude of non-gravitational part (matter plus vacuum). But, mathematical proof of zeroenergy universe needs the flatness of space as an assumption [4] i.e. zero-energy universe is a consequence of flatness. Since the space became flat only after inflation, the question remains about the source of energy before and during inflation. But, in this paper, we will prove zeroenergy universe without using flatness of space and so, energy can be conserved right from the beginning of the universe. In our model, flatness is actually a consequence of zero net energy density in the universe.

Of course one can argue, in an expanding space-time, Einstein's field equation doesn't imply conservation of energy due to matter and radiation [5]. This happens because Einstein's theory of gravity is a geometric theory and it considers only matter and radiation in its energy-momentum tensor. But, if we bring into the picture the concept of gravitational potential energy and are able to develop a cosmological model that satisfies the law of conservation of energy, the model will be scientifically more satisfying.

\section{Flatness and Horizon problems and inflationary solution:}

Infinitesimal length of a line element in our space-time is given by the generalized Friedmann-Robertson-Walker (FRW) metric,

$$
d \sigma^{2}=-c^{2} d t^{2}+R^{2}\left[\frac{d r^{2}}{1-k r^{2}}+r^{2} d \theta^{2}+r^{2} \sin ^{2} \theta d \phi^{2}\right]
$$


Where ' $R$ ' is scale factor (or radius of curvature), $k$ is observed curvature constant which can be +1 for positive curvature, 0 for flat space or -1 for negative curvature. Note that $k$ can take any value $(+1,0$ or -1$)$ depending upon the total energy density and rate of expansion of the universe even if the universe is physically closed (like an observer freely falling in a gravitational field cannot detect the curvature of space-time). Thus, in addition to energymomentum tensor, dynamics of the observer plays a very important role in the curvature measured by him. Friedmann's solution of Einstein's field equation is given by [6-7],

$$
\begin{aligned}
& \left(\frac{\dot{R}}{R}\right)^{2}=\frac{8 \pi G}{3} \rho-\frac{k}{R^{2}} c^{2} \\
& \frac{\ddot{R}}{R}=-\frac{4 \pi G}{3}\left(\rho+3 \frac{p}{c^{2}}\right)
\end{aligned}
$$

Critical density is defined by the dynamical properties of the universe is, $\rho_{\text {crit }}=\frac{3 H^{2}}{8 \pi G}$ where $H=\frac{\dot{R}}{R}$ (Hubble's constant). Energy density is expressed in terms of this critical density as $\Omega$-parameter defined by, $\Omega=\frac{\rho}{\rho_{\text {crit }}}$. Thus, in terms of above definitions, Eq. (2) becomes,

$$
\left(\Omega^{-1}-1\right) \rho R^{2}=\frac{-3 k c^{2}}{8 \pi G}
$$

Only if $\Omega$ is exactly equal to 1 , then $k=0$. If $\Omega>1, k=+1$. If $\Omega<1, k=-1$.

As in a matter dominated universe, $\rho$ varies as $R^{-3}$, Eq. (4) indicates that deviation of $\Omega$ from 1 is expected to increase in direct proportion to $R$. This instability of $\Omega$ near the value of $\sim 1$ is known as Flatness problem. To be specific, presently $\Omega$ is found to be close to 1 with an accuracy of better than $0.5 \%$. As R has increased by a factor of $\sim 10^{61}$ (from Planck size $\sim 10^{-35}$ $\mathrm{m}$ to current size $\sim 10^{26} \mathrm{~m}$ ), $\Omega$ must be close to 1 in the initial time with an accuracy of better than $10^{-62} \%$. Thus, flatness problem seeks an answer for such a precise fine tuning of $\Omega$ at the initial stage of the universe.

Horizon problem poses a challenge to explain the extraordinary homogeneity observed in temperature of causally disconnected regions of the universe despite of the finite speed of light. With expansion of the universe, temperature of the universe decreased and at about $\sim 380,000$ years, temperature was sufficiently low for recombination of electrons and protons and thus neutral atoms were formed. As neutral atoms have very low cross section for scattering of photons as compared to charged particles, photons decoupled from the plasma 
and have been streaming through the universe since that time. Of course, due to expansion of the universe, their wavelengths have been stretched and these photons are known as Cosmic Microwave Background (CMB) radiation. When we measure the spectrum of this $\mathrm{CMB}$ radiation, it very well fits to a black body spectrum of temperature of $\sim 2.726 \mathrm{~K}$. Even if we compare the temperatures of two opposite regions of the sky by their CMB radiation spectrum, both the temperatures agree with each other within an accuracy of $\sim 0.001 \mathrm{~K}$. The fact that we are measuring 'now' indicates, the two opposite regions have just come in causal contact with us. So, they must not have been in causal contact with each other as radiation from one part has covered only half the distance from other part. In fact, following the method of [8], it can be shown that the observable universe consists of about 36000 causally disconnected patches which surprisingly have almost same temperatures. This is as follows. If the present radius of the universe in form of 3 -sphere is $R_{0}$, the co-moving particle horizon at any time $t$ is given by,

$$
S(t)=R_{0} \int_{0}^{t} \frac{c d t}{R}
$$

Taking $R=A t^{\alpha}$ (for matter dominated universe $\alpha=2 / 3$ and for radiation dominated $\alpha=1 / 2$ ),

$$
S(t)=R_{0} \int_{0}^{t} \frac{c d t}{A t^{\alpha}}=\frac{R_{0} c t^{1-\alpha}}{A(1-\alpha)} \quad(\text { for } \alpha \neq 1)
$$

If $t$ is the time when photon decoupling occurred releasing the CMB radiation and $t_{0}$ is present time, the ratio of co-moving horizon volume today to the co-moving horizon volume at photon decoupling is,

$$
\begin{array}{ll} 
& {\left[\frac{S\left(t_{0}\right)}{S(t)}\right]^{3}=\left(\frac{t_{0}}{t}\right)^{3-3 \alpha}} \\
\text { Or } \quad & {\left[\frac{S\left(t_{0}\right)}{S(t)}\right]^{3}=\left(\frac{R_{0}}{R}\right)^{\frac{3}{\alpha}-3}=(1+z)^{\frac{3}{\alpha}-3}}
\end{array}
$$

Where $z=$ Red shift of $C M B$ radiation.

Taking $z=1100$ and $\alpha=2 / 3$ for matter dominated universe,

$$
\left[\frac{S\left(t_{0}\right)}{S(t)}\right]^{3}=36482
$$

Thus, presently observable universe contains about $\sim 36000$ causally disconnected patches. Still, they have almost same temperature of $\sim 2.726 \pm 0.001 \mathrm{~K}$. This is known as Horizon problem. 
In order to explain the Flatness and Horizon problems, Alan Guth [9-10] and others have proposed the cosmic inflation theory which assumes the existence of an inflaton field having a large potential energy in the early universe. Its negative pressure caused exponential expansion of the universe from $\sim 10^{-30} \mathrm{~m}$ to $\sim 10^{-2} \mathrm{~m}$ during a period from about $\sim 10^{-35} \mathrm{~s}$ to $\sim$ $10^{-32} \mathrm{~s}$ after creation of the universe. Since the energy density of the inflaton field is assumed to be nearly constant during this expansion, Eq. (4) indicates that whatever initial value $\Omega$ starts with, it approaches 1 with increasing radius $R$ (as deviation from 1 is proportional to $R^{-}$ ${ }^{2}$ ). Thus, instability of $\Omega$ is avoided during early stage of universe. After $\sim 10^{-32} \mathrm{~s}$ even if inflation stops, $\Omega$ is already so close to 1 that it will take very long to deviate from 1 . This is how inflation theory explains the avoidance of instability of $\Omega$ in early universe and why the value of $\Omega$ is $\sim 1$. Similarly, as the universe expanded by a huge factor of $\sim 10^{28}$ in a very small time interval of $\sim 10^{-32} \mathrm{~s}$, outer portion of every causal patch was pushed out of the horizon. After inflation stopped and as horizon still expands at the speed of light, we are seeing those parts which were originally in causal contact with us before inflation. Thus, horizon problem is solved in cosmic inflation theory.

But, the limitation of the inflation theory is that it cannot explain the present rate of expansion of the universe by predicting the value of vacuum energy density and it also can't predict matter energy density. Since the space became flat only after inflation, inflationary model can't prove the net energy of universe to be zero before inflation and thus the law of energy conservation is violated during pre-inflation period. In addition, the magnitude and variation of the potential energy with strength of the inflaton field are artificially decided to make it fit to explain the flatness and horizon problems. That's why currently there are so many inflationary models proposed in literature. Mechanism of graceful exit from inflationary stage also casts some doubt on the theory. Recently due to this, Paul J. Steinhardt, one of the architects of the theory, has turned against it [11-12].

\section{Our approach:}

To solve the above mentioned problems in an unified manner, we will develop a generalized version of our recent cosmological model [13] where we derived the expression to calculate the density of vacuum energy that needs only Hubble's constant as input (whereas standard cosmology needs both Hubble's constant and matter density). We had also developed expression for energy density of matter component for which standard cosmology has no predictive power. However, earlier we had taken some assumptions which led to 
approximate results. In this paper, we will remove those assumptions and develop a more generalized version of cosmological model that explains how creation of universe doesn't violate the law of conservation of energy and also simultaneously solves the cosmological constant problem, cosmic coincidence problem, flatness problem and horizon problem of the universe.

The structure of the paper is as follows. In the next section, we describe our improved version of cosmological model that leads to expression for vacuum and matter energy density and solves cosmic coincidence problem. Using these expressions, in the next three sections, we demonstrate working of the law of conservation of energy and then solve the cosmological constant, flatness and horizon problems in our model. Lastly, in the conclusion, we present a table comparing the advantages and predictive power of our cosmological model with that of the standard model.

\section{Development of generalized cosmological model and solution of cosmic coincidence problem}

In standard cosmology, universe is assumed to be isotropic and homogeneous. To impose the condition of isotropy, Einstein visualized the universe as a closed hyper-surface (surface of a 3-sphere) existing in a 4-dimensional space. This exciting idea of Einstein as expressed in his original paper [14] is quoted below.

"We arrive at such a space, for example, in the following way. We start from a Euclidean space of four dimensions, $\xi_{1}, \xi_{2}, \xi_{3}, \xi_{4}$, with a linear element $d \sigma$; let therefore, $d \sigma^{2}=d \xi_{1}^{2}+d \xi_{2}^{2}+d \xi_{3}^{2}+d \xi_{4}^{2}$

In this space we consider the hyper-surface, $R^{2}=\xi_{1}^{2}+\xi_{2}^{2}+\xi_{3}^{2}+\xi_{4}^{2}$

Where $R$ denotes a constant. The points of this hyper-surface form a threedimensional continuum, a spherical space of radius of curvature R."

To maintain isotropy of space, we will follow the Einstein's model of universe that lies on the surface of a 3 -sphere (hyper-sphere) existing in a four-dimensional space. As described in section-1, curvature constant $k$ in FRW metric of this space represents the observed characteristics of a line element depending upon energy density and Hubble's constant. So, even if globally the universe is closed and finite, $k$ may be $+1,0$ or -1 depending upon the rate of expansion of universe (just like space-time looks flat to an observer freely falling under a gravitational field). As per quantum field theory, this space in the form of hypersurface of 3-sphere must have the zero-point energy and sum of energies for all possible 
modes of oscillations is the vacuum energy. We will assume a common zero-point scalar field that is responsible for energy of all the fundamental particles. Number of particles having same energy increases when a particular zero-point mode is excited. Properties of particles other than energy appear when excited field couples with other fields such as Higg's field, electromagnetic field etc. So, particles of same energy even if have different rest mass, charge or spin correspond to a single and common mode of zero-point wave.

Since the space of our universe is closed and spherically symmetric, circumference must be integer multiple of wavelength of zero-point wave. Mathematically,

$$
2 \pi R=n \lambda
$$

Where $R$ is the radius of universe in the form of 3-sphere, $\lambda$ is wavelength of zero-point field, $n$ is an integer varying from 1 to $n_{\max }$ and $n_{\max }$ is decided by lowest possible wavelength $\lambda_{\min }$. Minimum wave length must be of the order of Planck length $l_{p}=\sqrt{\frac{\hbar G}{c^{3}}}=1.6 \times 10^{-35} \mathrm{~m}$, as distances smaller than Planck length cannot be defined [15-16]. We will take the cutoff wavelength as $\lambda_{\min }=\beta l_{p}$ where $\beta$ is a constant nearly equal to 1 . Using this $\lambda_{\min }$ in Eq. (7) we get,

$$
n_{\max }=\frac{2 \pi R}{\lambda_{\min }}=\frac{2 \pi R}{\beta l_{p}}
$$

Initial radius $R_{i}$ of universe can be found from Eq. (8) by putting $n_{\max }$ equal to 1 and it is given by,

$$
R_{i}=\frac{\beta l_{p}}{2 \pi} .
$$

Let us now consider the example of a particle trapped in a one-dimensional box of size $L$. Although its magnitude of momentum is quantized by, $p=\frac{n h}{2 L}$, the direction of momentum remains in a state of superposition of $+\mathrm{ve}$ and $-\mathrm{ve}$ directions along $\mathrm{X}$-axis due to symmetry. Similarly, consider the Bohr's hydrogen atom. An electron while revolving around the nucleus remains as a standing wave and we only quantize the wavelength or magnitude of momentum. The direction of momentum of the electron in Bohr's theory remains in a state of superposition of all possible directions in 3-D space due to spherical symmetry. We don't consider a separate state corresponding to each possible direction of momentum. Similarly, for the standing zero-point scalar wave on the surface of hyper-sphere of universe, direction of momentum can remain in a state of superposition of all possible directions due to 
symmetry. Zero-point scalar field can continue to exist in a superposition state as it is unable to interact with anything else. That's why, in contrast to conventional method, we will consider only the modes with distinct magnitudes of momentum. Different directions of momentum cannot lead to new modes as each zero-point scalar wave propagates simultaneously in a quantum superposition state along all directions in 3-D space. So, total vacuum energy of the universe considering all modes of zero-point oscillations of different magnitudes of momentum is given by,

$$
U_{v a c}=\sum \frac{1}{2} h v=\frac{h c}{2} \sum \frac{1}{\lambda}
$$

Using Eq. (7),

$$
U_{v a c}=\frac{h c}{4 \pi R} \sum_{1}^{n_{\max }} n=\frac{\hbar c}{4 R} n_{\max }^{2}\left(1+\frac{1}{n_{\max }}\right)
$$

Putting the value of $n_{\max }$ from Eq. (8) and $l_{p}=\sqrt{\frac{\hbar G}{c^{3}}}$ in above expression, total vacuum energy becomes,

$$
U_{v a c}=\frac{\pi^{2} c^{4} R}{\beta^{2} G}\left(1+\frac{\beta l_{p}}{2 \pi R}\right)
$$

Dividing the above by the surface volume of hyper-sphere $2 \pi^{2} R^{3}$, vacuum energy density is,

$$
u_{v a c}=\frac{c^{4}}{2 \beta^{2} G R^{2}}\left(1+\frac{R_{i}}{R}\right)
$$

As $R \gg R_{i}$, second term in (10) and (11) is negligible and so, vacuum energy and its density are given by,

$$
\begin{aligned}
& U_{v a c}=\frac{\pi^{2} c^{4} R}{\beta^{2} G} \\
& u_{v a c}=\rho_{v a c} c^{2}=\frac{c^{4}}{2 \beta^{2} G R^{2}}
\end{aligned}
$$

Numerical value of vacuum energy density $u_{v a c}$ will be calculated in section- 4 after we derive the analytical expression for $R$.

Now, Let us calculate the average energy density due to non-vacuum components (ordinary matter, dark matter and radiation) of the universe. If $\phi$ is scalar field of the zeropoint oscillation, energy density and pressure are given by [17], 


$$
\begin{aligned}
& u_{v a c}=\frac{1}{2}\left(\frac{\partial \phi}{c \partial t}\right)^{2}+\frac{1}{2}\left[\left(\frac{\partial \phi}{\partial x}\right)^{2}+\left(\frac{\partial \phi}{\partial y}\right)^{2}+\left(\frac{\partial \phi}{\partial z}\right)^{2}\right]+V(\phi) \\
& p=\frac{1}{2}\left(\frac{\partial \phi}{c \partial t}\right)^{2}-\frac{1}{6}\left[\left(\frac{\partial \phi}{\partial x}\right)^{2}+\left(\frac{\partial \phi}{\partial y}\right)^{2}+\left(\frac{\partial \phi}{\partial z}\right)^{2}\right]-V(\phi)
\end{aligned}
$$

Just like in a harmonic oscillator, energy will be shared equally between potential energy (depending on $\phi$ ) and kinetic energy (depending on derivatives of $\phi$ ). So,

$$
\frac{1}{2}\left(\frac{\partial \phi}{c \partial t}\right)^{2}+\frac{1}{2}\left[\left(\frac{\partial \phi}{\partial x}\right)^{2}+\left(\frac{\partial \phi}{\partial y}\right)^{2}+\left(\frac{\partial \phi}{\partial z}\right)^{2}\right]=V(\phi)
$$

Due to relativistic consideration, giving equal status to time and space coordinates, each term in LHS of Eq. (16) becomes equal. So,

$$
\frac{1}{2}\left(\frac{\partial \phi}{c \partial t}\right)^{2}=\frac{1}{2}\left(\frac{\partial \phi}{\partial x}\right)^{2}=\frac{1}{2}\left(\frac{\partial \phi}{\partial y}\right)^{2}=\frac{1}{2}\left(\frac{\partial \phi}{\partial z}\right)^{2}=\frac{V(\phi)}{4}
$$

Using Eq. (16) and (17) in Eq. (14) and (15), we get,

$$
\begin{aligned}
& u_{v a c}=2 V(\phi), \quad p=-V(\phi) \\
& \Rightarrow p=-\frac{u_{v a c}}{2}
\end{aligned}
$$

Expanding the bracket in Eq. (10) and then taking differential,

$$
d U_{v a c}=\frac{\pi^{2} c^{4}}{\beta^{2} G} d R
$$

In a matter dominated universe, pressure is mainly caused by vacuum energy (gravitational potential energy and its pressure are not considered in Einstein field equation as it is a geometric theory and pressure due to radiation is neglected as radiation energy is less than $\sim 0.1 \%$ ). Since Einstein's field equation makes the covariant derivative of energy-momentum tensor zero $\left(T_{; v}^{\mu v}=0\right)$, energy conservation equation as derived by Weinberg [18] is,

$$
\begin{gathered}
d\left(u R^{3}\right)+p\left(3 R^{2} d R\right)=0 \\
d U_{\text {nonvac }}+d U_{\text {vac }}+p d V=0
\end{gathered}
$$

(As surface volume of hyper-sphere $V=2 \pi^{2} R^{3}$ )

Using Eq. (19), (18) and (11) in Eq. (20), we get,

$$
d U_{\text {nonvac }}=-\frac{\pi^{2} c^{4}}{\beta^{2} G} d R+\frac{3 \pi^{2} c^{4}}{2 \beta^{2} G}\left(1+\frac{R_{i}}{R}\right) d R
$$




$$
\text { Or } \quad d U_{\text {nonvac }}=\frac{\pi^{2} c^{4}}{2 \beta^{2} G}\left(1+3 \frac{R_{i}}{R}\right) d R
$$

Thus, we find that non-vacuum energy of the universe also increases with radius of the universe. Increase of non-vacuum energy happens by way of excitation of zero-point oscillators (i.e. creation of matter and radiation). We will prove in next section that the energy required for increase of both vacuum and non-vacuum parts comes from gravitational potential energy which becomes more and more negative and keeps the net energy of universe zero.

Integrating Eq. (21) from $R_{i}$ to $R$, we get,

$$
U_{\text {nonvac }}=\frac{\pi^{2} c^{4} R}{2 \beta^{2} G}\left[1-\frac{R_{i}}{R}+\frac{3 R_{i}}{R} \ln \left(\frac{R}{R_{i}}\right)\right]
$$

Dividing Eq. (22) by surface volume $\left(2 \pi^{2} R^{3}\right)$ of hyper-sphere, we get of non-vacuum energy density,

$$
u_{\text {nonvac }}=\frac{C^{4}}{4 \beta^{2} G R^{2}}\left[1-\frac{R_{i}}{R}+\frac{3 R_{i}}{R} \ln \left(\frac{R}{R_{i}}\right)\right]
$$

For $R>>R_{i}$

$$
\begin{aligned}
& U_{\text {nonvac }}=\frac{\pi^{2} c^{4} R}{2 \beta^{2} G} \\
& u_{\text {nonvac }}=\rho_{\text {nonvac }} c^{2}=\frac{c^{4}}{4 \beta^{2} G R^{2}}
\end{aligned}
$$

If $R>>10^{-35} \mathrm{~m}$, comparing Eq. (13) and (25), we find that the ratio of non-vacuum energy density to the vacuum energy density is $1 / 2$ and it is independent of radius $R$ of universe and in fact independent of all constants of the universe. In other words, theoretically, $\sim 66.7 \%$ of total energy should be in the form of dark energy and rest $33.3 \%$ should be in the form of non-vacuum component i.e. matter, dark matter and radiation since the beginning of universe. Thus, the cosmic coincidence problem which questions why the matter energy density is of the same order as vacuum energy density is explained.

\section{Conservation of energy in an expanding universe}

Using Eq. (13) and (25), total mass density due to vacuum and non-vacuum energy is given by,

$$
\rho=\rho_{\text {vac }}+\rho_{\text {nonvac }}=\frac{3 c^{2}}{4 \beta^{2} G R^{2}}
$$


Putting the above value $\rho=\frac{3 c^{2}}{4 \beta^{2} G R^{2}}$ and $p=-\frac{u_{v a c}}{2}=\frac{-c^{2}}{4 \beta^{2} G R^{2}}$ from Eq. (18) and Eq. (13) in Fiedmann's Equations (2) and (3), we get,

$$
\begin{aligned}
& \left(\frac{\dot{R}}{R}\right)^{2}=\frac{8 \pi G}{3}\left(\frac{3 c^{2}}{4 \beta^{2} G R^{2}}\right)-\frac{k}{R^{2}} c^{2} \\
& \frac{\ddot{R}}{R}=-\frac{4 \pi G}{3}\left(\frac{3 c^{2}}{4 \beta^{2} G R^{2}}-3 \frac{c^{2}}{4 \beta^{2} G R^{2}}\right)=0
\end{aligned}
$$

Simplifying,

$$
\dot{R}=c \sqrt{\frac{2 \pi}{\beta^{2}}-k}
$$

And

$$
\ddot{R}=0
$$

Thus, we find that the radius of universe increases with time at a uniform rate of $\dot{R}=\gamma c$, where $\gamma=\sqrt{\frac{2 \pi}{\beta^{2}}-k}$. So, at any instant of time $\tau$, radius of universe is, $R=R_{i}+\gamma c \tau$. After few time steps of Planck time $\left(\sim 10^{-44} \mathrm{~s}\right), R_{i}<<\gamma c \tau$ so that we can write, $R=\gamma c \tau$.

For calculation of gravitational potential energy of any small mass $m$ due another larger Mass $M$, we should use the general relativistic expression given by Voracek [19], P.E. $=m c^{2}\left[\left(1-\frac{2 G M}{c^{2} r}\right)^{1 / 2}-1\right]$. However, as the Schwarzschild radius is very much small as compared to interstellar/ intergalactic distances i.e. $\frac{2 G M}{c^{2}}<<r$, above expression reduces to,

$$
\text { P.E. }=m c^{2}\left[\left(1-\frac{1}{2} \frac{2 G M}{c^{2} r}\right)-1\right]=-\frac{G M m}{r}, \text { which is Newton's law. }
$$

So, we can safely use Newton's law for calculating gravitational potential energy. However, since gravitational effect is transmitted at the speed of light, we will use the retarded form of Newton's expression for gravitational potential energy and also consider time dependence of energy density of the universe. As shown in Fig. 1, mass at a specific time $\tau$ contained in thin strip on the surface of hyper-sphere at angular distance of $\phi$ from a unit mass can contribute to the gravitational potential energy at any time $t$ and distance to be used in Newton's expression must be $c(t-\tau)$. 


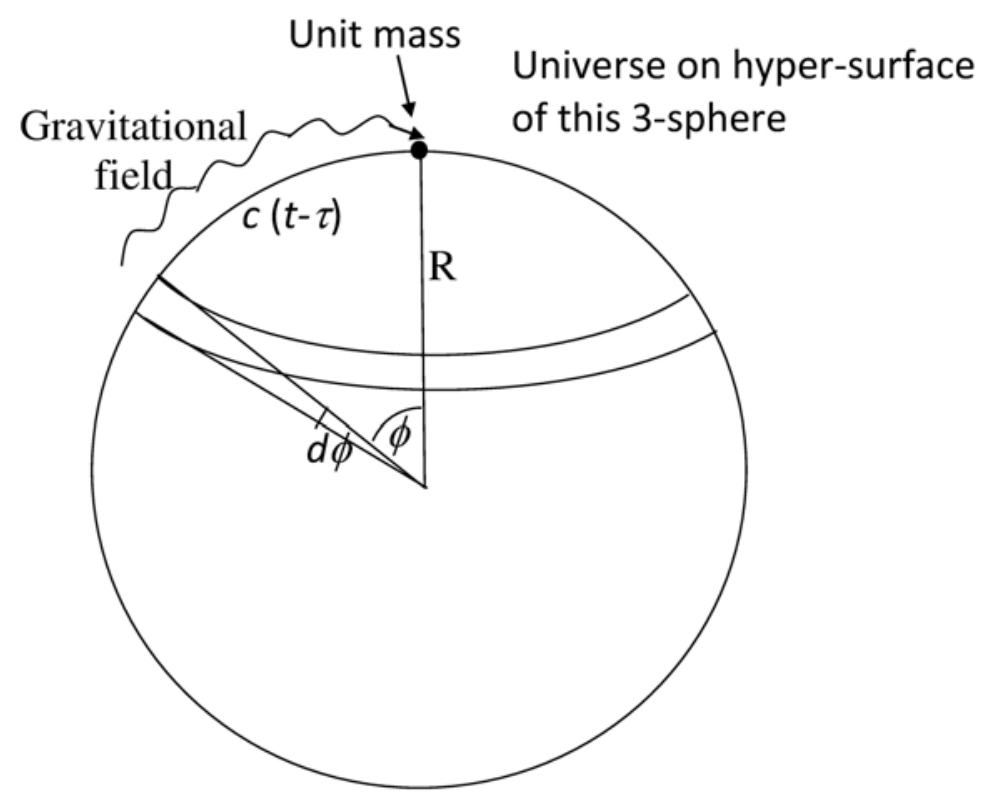

Fig. 1 Gravitational potential energy due to a thin strip equidistant from a unit mass

So, gravitational potential energy (P.E.) of the unit mass is,

$$
P . E .=-\int_{0}^{\phi_{\max }} G \frac{\rho(\tau) 4 \pi[R(\tau)]^{3} \sin ^{2} \phi d \phi}{c(t-\tau)}
$$

Another condition is that the $\phi$ must be equal to the angular distance covered by the gravitational radiation during the time interval from $\tau$ to $t$. Mathematically,

$$
\begin{aligned}
\phi & =\int_{\tau}^{t} \frac{c d \tau^{\prime}}{R}=\int_{\tau}^{t} \frac{c d \tau^{\prime}}{\gamma c \tau^{\prime}}=\frac{1}{\gamma} \ln \left(\frac{t}{\tau}\right) \\
\Rightarrow \quad t & =\tau e^{\phi \gamma}
\end{aligned}
$$

From Eq. (30), maximum limit of $\phi$ is, $\phi_{\max }=\infty$ (when $\tau=0$ ). Using the value of total mass density $\rho$ from Eq. (26) and value of $t$ from Eq. (30), Eq. (29) becomes,

$$
\text { P.E. }=-\frac{3 \pi c}{\beta^{2}} \int_{0}^{\infty} \frac{R \sin ^{2} \phi d \phi}{\tau\left(e^{\phi \gamma}-1\right)}
$$

As $R(\tau)=\gamma c \tau$, we get,

$$
\text { P.E. }=-\frac{3 \pi c^{2} \gamma}{\beta^{2}} \int_{0}^{\infty} \frac{\sin ^{2} \phi d \phi}{\left(e^{\phi \gamma}-1\right)}
$$

As $\gamma=\sqrt{\frac{2 \pi}{\beta^{2}}-k}$ 


$$
\text { P.E. }=-\frac{3 c^{2} \gamma\left(\gamma^{2}+k\right)}{2} \int_{0}^{\infty} \frac{\sin ^{2} \phi d \phi}{\left(e^{\phi \gamma}-1\right)}
$$

Total gravitational energy due to all the matter/vacuum of energy $U_{\text {matt }+v a c}$ is given by,

$$
U_{\text {grav }}=\frac{1}{2}(P . E .) \times \frac{U_{\text {matt }+v a c}}{c^{2}}
$$

(Note factor $1 / 2$ is here to avoid double counting of interaction energy between two particles) Using Eq. (31) in above,

$$
\frac{U_{\text {grav }}}{U_{\text {matt }+v a c}}=-\frac{3 \gamma\left(\gamma^{2}+k\right)}{4} \int_{0}^{\infty} \frac{\sin ^{2} \phi d \phi}{\left(e^{\phi \gamma}-1\right)}
$$

Evaluating above expression, we find that it can be equal to -1 (or $U_{\text {grav }}=-U_{\text {matt } v a c}$ ) only if $\gamma=2$ (for $k=0$ ), $\gamma=1.12$ (for $k=+1$ ) and $\gamma=2.5$ (for $k=-1$ ). Thus, whatever be the value of $k$, it is possible to have total energy of the universe, $U_{\text {Total }}=U_{\text {matt } v a c}+U_{\text {grav }}=0$ (Irrespective of the radius of universe). So, law of conservation of energy is not violated during the process of creation of the universe. It will be proved in next section that $k=0(\gamma=2)$ is the only possible value from general relativistic consideration.

\section{Solution of Flatness problem and Cosmological constant problem}

We first restate the Eq. (4) i.e. Friedmann's first equation in terms of $\Omega$,

$$
\left(\Omega^{-1}-1\right) \rho R^{2}=\frac{-3 k c^{2}}{8 \pi G}
$$

Putting the value $\rho=\frac{3 c^{2}}{4 \beta^{2} G R^{2}}$ from Eq. (26), we get

$$
\left(\Omega^{-1}-1\right)=\frac{-k \beta^{2}}{2 \pi}
$$

Thus, as deviation of $\Omega$ from 1 remains constant irrespective of the radius of the universe, the instability of $\Omega$ (which is main concern for flatness problem) is eliminated. However, as $\Omega$ is a constant as per Eq. (33), for three possible values of $k=+1,0$ and -1 , there are three possible values of $\Omega$. We will now predict the expected value of $\Omega$ which will be proved to be equal to 1 .

In the previous section we have proved that law of energy conservation can be satisfied for specific pairs of values of $\gamma$ and $k$. When it happens, gravitational potential 
energy density $u_{\text {grav }}$ becomes equal and opposite of the average non-gravitational energy density $u_{\text {matt }+v a c}$ (matter plus vacuum). So, net energy density, $u_{\text {grav }}+u_{\text {matt }+v a c}=0$. Since gravitational field moves at the speed of light, its pressure is given by, $p_{\text {grav }}=\frac{1}{3}\left|u_{\text {grav }}\right|$ (modulus is taken as pressure due to gravitational radiation is positive). As described in section 2, out of the non-gravitational part, pressure is caused by vacuum energy and it is negative given by, $p=-\frac{u_{v a c}}{2}$ and $u_{v a c}=\frac{2}{3} u_{\text {matt } v a c}$. So, $p=-\frac{u_{\text {matt }+v a c}}{3}$. So net pressure is given by, $\quad p_{\text {net }}=p_{\text {grav }}+p=\frac{1}{3}\left|u_{\text {grav }}\right|-\frac{u_{\text {matt }+v a c}}{3}=0 \quad$ (as magnitudes of $u_{\text {grav }}$ and $u_{\text {matt }+v a c}$ are equal). Thus, total energy density and total pressure become zero all over the universe.

Now, let us write the linearized form of Einstein field equation as derived by Weinberg [18],

$$
R_{\mu \nu}^{(1)}-\frac{1}{2} \eta_{\mu \nu} R_{\lambda}^{(1) \lambda}=\frac{8 \pi G}{c^{4}}\left[T_{\mu \nu}+t_{\mu \nu}\right]
$$

Second term in right hand side of above equation represents the contribution due to gravitational field in the total energy-momentum tensor. In our case, we have seen that contributions of gravitational field make both total energy density and pressure equal to zero. Thus, as the right hand side of Eq. (34) i.e. total energy-momentum pseudo-tensor becomes zero all over the space of the universe, curvature of space-time also becomes zero i.e. $k=0$.

Using $k=0$ in Eq. (33), $\Omega=1$ or space is always flat.

Putting $k=0$ in $\gamma=\sqrt{\frac{2 \pi}{\beta^{2}}-k}$, we get,

$$
\gamma=\frac{\sqrt{2 \pi}}{\beta}
$$

Since Hubble's constant is $H=\frac{\dot{R}}{R}$,

Radius of universe is, $R=\frac{\dot{R}}{H}=\frac{\gamma c}{H}=\frac{\sqrt{2 \pi} c}{\beta H}$

Putting the above expression of $R$ in Eq. (13) and (25), vacuum energy density and nonvacuum energy density become,

$$
\begin{aligned}
& u_{v a c}=\frac{c^{2} H^{2}}{4 \pi G} \\
& u_{\text {nonvac }}=\frac{c^{2} H^{2}}{8 \pi G}
\end{aligned}
$$


Note that, in contrast to standard cosmology, vacuum energy density here needs only the Hubble's constant and doesn't need matter density for its calculation. The value of $u_{v a c}$ calculated from Eq. (36) is $\sim 5.2 \times 10^{-10} \mathrm{~J} / \mathrm{m}^{3}$ (taking $H=67.9 \mathrm{~km} / \mathrm{s} \mathrm{Mpc}^{-1}$ ) and it agrees well with the value estimated using standard cosmological model and observed data of Planck Collaboration [20]. The earlier difference by a factor of $\sim 10^{123}$ between quantum mechanical calculation and cosmological observation of vacuum energy is eliminated and cosmological constant problem is solved. Non-vacuum energy density $u_{\text {nonvac }}$ (which standard cosmology can't predict) can be estimated from Eq. (37) and it is found to be $\sim 2.6 \times 10^{-10}$ $\mathrm{J} / \mathrm{m}^{3}$. It matches well with the observed data of Planck Collaboration [20].

Finally, using the conclusion of previous section i.e. $\gamma=2$ (corresponding to $k=0$ ) for energy conservation, rate of expansion of universe is, $\dot{R}=\gamma c=2 c$. As $\gamma=\sqrt{2 \pi} / \beta, \beta=1.25$. So, minimum possible wavelength of a zero-point wave is 1.25 times the Planck length. Initial radius $R_{i}$ of universe is given by, $R_{i}=\frac{\beta l_{p}}{2 \pi}=3.2 \times 10^{-36} \mathrm{~m}$. Because universe expands at a uniform rate of $\dot{R}=2 c$, age of universe is, $t_{0}=R_{0} / \dot{R}=1 / H_{0}=14.4$ billion years. As angular distance of any galaxy from us on the surface of 3-sphere remains constant during expansion of the universe, if its distance from us is $x, \dot{x} / x=\dot{R} / R \Rightarrow \dot{x}=H x$ (Hubble's law). At present time, Hubble's constant $\left(H_{0}=1 / t_{0}\right)$ remains nearly constant during our period of measurement. So, acceleration of any galaxy from us is $\ddot{x}=H_{0} \dot{x}=H_{0}{ }^{2} X \neq 0$ even though $\ddot{R}=0$ as per Eq. (28). Physically, a star appears accelerating from us because farther it goes, more space is there in between to expand.

\section{Solution of Horizon problem:}

As the rate of expansion of universe is constant i.e. $\dot{R}=2 c$, we can write, $R=R_{i}+2 c t$ (neglecting nonlinear part during the time comparable to Planck time $\sim 10^{-44} \mathrm{~s}$ ). So, for $R>>R_{i}, \alpha=1$. If we put $\alpha \rightarrow 1$ in Eq. (6), number of causally disconnected patches comes out to be 1 (not 36000) and horizon problem is solved. However, we will not directly use eq. (6) as it assumes $R=A t^{\alpha}$ and $\alpha \neq 1$. So, putting $R=R_{i}+2 c t$ in Eq. (5), co-moving particle horizon at any time $t$ is given by,

$$
S(t)=R_{0} \int_{0}^{t} \frac{c d t}{R_{i}+2 c t}=\frac{R_{0}}{2} \ln \left[1+\frac{2 c t}{R_{i}}\right]
$$


Then, the ratio of co-moving horizon volume at present time $t_{0}$ to the co-moving horizon volume at the time $t_{C M B}$ of photon decoupling is,

$$
\left[\frac{S\left(t_{0}\right)}{S\left(t_{C M B}\right)}\right]^{3}=\left(\ln \left[1+\frac{2 c t_{0}}{R_{i}}\right] / \ln \left[1+\frac{2 c t_{C M B}}{R_{i}}\right]\right)^{3}
$$

Putting $R_{i}=3.2 \times 10^{-36} \mathrm{~m}, t_{0}=14.4$ by and $t_{C M B}=380000 \mathrm{y}$,

$$
\left[\frac{S\left(t_{0}\right)}{S\left(t_{C M B}\right)}\right]^{3}=1.24
$$

Thus, we find that, in contrast to standard cosmology, the whole observable universe is nearly causally connected and it doesn't contain $\sim 36000$ causally disconnected patches.

We will also give here an alternative calculation which will show that the co-moving size of patch from which $\mathrm{CMB}$ photons come to us is much smaller than the co-moving particle distance at the time of release of CMB radiation. After the release of CMB radiation, the photons we are detecting now must have moved through an angular distance of,

$$
\begin{gathered}
\theta=\int_{t_{C M B}}^{t_{0}} \frac{c d t}{R}=\int_{t_{C M B}}^{t_{0}} \frac{c d t}{R_{i}+2 c t} \\
\theta=\frac{1}{2} \ln \left(\frac{R_{i}+2 c t_{0}}{R_{i}+2 c t_{C M B}}\right)
\end{gathered}
$$

Putting $t_{0}=14.4$ by and $t_{C M B}=380,000 y, \theta=5.27$ radian. Since, this is more than $\pi$, as shown Fig. 2, minimum angular distance between two opposite points $\mathrm{A}$ and $\mathrm{B}$ of our present horizon is, $\varphi=2(2 \pi-\theta)=2.03 \mathrm{rad}$.

Theoretically maximum angular distance a photon can move till the time of photon decoupling is,

$$
\psi=\int_{0}^{t_{C M B}} \frac{c d t}{R}=\int_{0}^{t_{C M B}} \frac{c d t}{R_{i}+2 c t}=\frac{1}{2} \ln \left[1+\frac{2 c t_{C M B}}{R_{i}}\right]
$$

Putting $t_{C M B}=380,000 \mathrm{yr}$ and $R_{i}=3.2 \times 10^{-36} \mathrm{~m}$,

$$
\psi=66 \text { radian }
$$




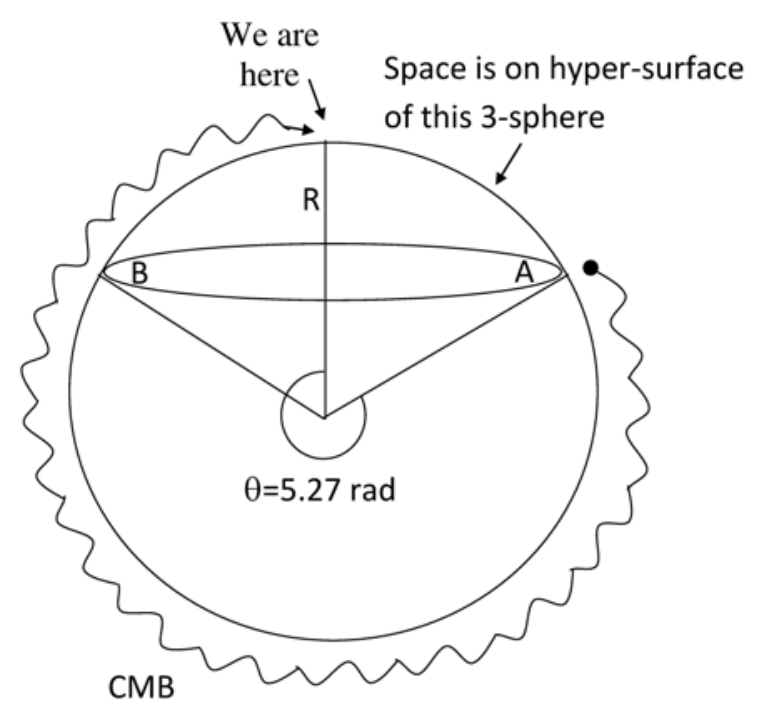

Fig.2 Angular distance between two opposite points A and B on our horizon located on the hyper-surface of 3-sphere

Thus, as $\frac{\psi}{\varphi}=33$ i.e. maximum angular distance that a photon can move till CMB release is about $\sim 33$ times more than the angular distance between two opposite points of horizon, it is possible for our horizon to attain thermal equilibrium and achieve nearly same temperature. Thus, our cosmic model solves the horizon problem.

\section{Conclusion}

In this paper, we have developed a unified cosmological model to solve five important astrophysical problems viz. cosmological constant problem, cosmic coincidence problem, energy conservation, flatness and horizon problem. Our model predicts the density of vacuum energy taking only Hubble's constant as input whereas standard cosmology needs both Hubble's constant and matter density to estimate vacuum energy density. We have also developed expression for energy density of non-vacuum component (matter pus radiation) which standard cosmology can't predict. In contrast to standard inflationary model, our theory doesn't need flatness of space as a pre-requirement to prove zero-energy universe or to save law of conservation of energy. In our model, flatness of space is a consequence of zeroenergy universe. We have found that total vacuum and non-vacuum energy increases with expansion of the universe and it comes from gravitational potential energy which becomes more and more negative. In contrast to standard cosmology, our model doesn't have to invoke any inflaton field having a specific potential energy distribution to prove flatness and 
homogeneity of horizon. It just uses quantized zero point scalar field in a closed universe. Highlights of the advantages and predictive power of our cosmological model as compared to standard model are given in Table. 1.

At present, standard $\Lambda \mathrm{CDM}$ model is used to infer evolution of temperature and density of universe from CMB spectra and anisotropy. But, if we take same CMB spectra and anisotropy data and apply our new cosmological model, we expect to reach at somewhat different conclusions about temperature and density of early universe. This part along with nucleosynthesis and galaxy formation will be investigated in future.

Table. 1 A comparison of advantages and predictive power of our new cosmological model with those of standard model

\begin{tabular}{|c|c|c|}
\hline $\begin{array}{c}\text { Variables } \\
\text { to be determined }\end{array}$ & Standard inflationary cosmology & New cosmological model \\
\hline Vacuum energy density & $\begin{array}{c}\text { To predict, needs } \\
\text { both Hubble's constant } \\
\text { and Matter density }\end{array}$ & $\begin{array}{c}\text { To predict, needs } \\
\text { only Hubble's constant }\end{array}$ \\
\hline $\begin{array}{c}\text { Non-vacuum energy } \\
\text { density }\end{array}$ & Cannot predict & $\begin{array}{c}\text { To predict, needs } \\
\text { only Hubble's constant }\end{array}$ \\
\hline $\begin{array}{c}\text { Ratio of vacuum } \\
\text { energy to total energy }\end{array}$ & $\begin{array}{c}\text { Needs Hubble's constant and } \\
\text { Matter density }\end{array}$ & $\begin{array}{c}\text { No input required. } \\
\text { Ratio=2/3=66.7\% }\end{array}$ \\
\hline $\begin{array}{c}\text { Is energy conserved } \\
\text { considering } \\
\text { gravitational potential } \\
\text { energy? }\end{array}$ & $\begin{array}{c}\text { Yes } \\
\text { inflation or space made flat) }\end{array}$ & $\begin{array}{c}\text { Flatness is a consequence } \\
\text { of energy conservation }\end{array}$ \\
\hline $\begin{array}{c}\text { To explain Flatness of } \\
\text { space }\end{array}$ & $\begin{array}{c}\text { Needs Inflaton field with a specific } \\
\text { potential energy distribution }\end{array}$ & $\begin{array}{c}\text { Needs only } \\
\text { Zero-point field }\end{array}$ \\
\hline $\begin{array}{c}\text { To explain } \\
\text { homogeneity of } \\
\text { Horizon }\end{array}$ & $\begin{array}{c}\text { Needs Inflaton field with a specific } \\
\text { potential energy distribution }\end{array}$ & $\begin{array}{c}\text { Needs only } \\
\text { Zero-point field }\end{array}$ \\
\hline
\end{tabular}

\section{Acknowledgements}

Author is very much thankful to Prof. James D Stein, Emeritus Professor, California State University, USA for his thorough reading of the manuscript and useful suggestions. Author is grateful to Prof. Archana Sharma, Associate Director, BTDG, Bhabha Atomic Research Centre, India for her encouragement and support during this research. 


\section{References}

[1] Steven Weinberg, "The cosmological constant problem", Reviews of Modern Physics 1989; 61 (1): 1-23

[2] Thomas Banks, “The Cosmological Constant Problem”, Physics Today, 2004; 57( 3): 46-51

[3] Velten H. E. S., Marttens R. F. vom and Zimdahl W., "Aspects of the cosmological "coincidence problem"”, The European physical Journal C, 2014; 74:3160, 1-8

[4] Lawrence M. Krauss, "A universe from nothing” (Free Press, New York), 2012

[5] Sean Carroll, "Energy is not conserved", Discover, 2010, February 23

[6] Sean M Carroll, "The Cosmological Constant", Living Rev. Relativity, 2001; 4(1): 1-56

[7] Friedmann A., "Uber die Krummung des Raumes", Zeitschrift fur Physik, 1922; 10: 377386. (English version) "On the Curvature of Space", General Relativity and Gravitation, 1999; 31(12):1991-2000

[8] David H. Weinberg, "Inflation", Lecture Notes, Department of Astronomy, Ohio State University; 2020 (www.astronomy.ohio-state.edu/ dhw/A873/notes7.pdf)

[9] Alan H. Guth, "Infiationary universe: A possible solution to the horizon and flatness problems", Physical Review D, 1981; 23(2): 347-356

[10] Alan Guth, "Was cosmic inflation the 'bang' of the big bang?", Beam Line, 1997; 27(3):14-21 (https://ned.ipac.caltech.edu/level5/Guth/Guth_contents.html)

[11] Anna Ijjas, Steinhardt Paul J. and Loeb Abraham, "Inflationary paradigm in trouble after Planck 2013”, Physics Letters B, 2013; 723 (4-5): 261-266

[12] Anna Ijjas, Steinhardt Paul J. and Loeb Abraham, "Inflationary schism”, Physics Letters B, 2014; 736:142-146

[13]Biswaranjan Dikshit, "Quantum Mechanical Explanation for Dark Energy, Cosmic Coincidence, Flatness, Age, and Size of the Universe", Open Astronomy, 2019; 28: 220227

[14] A. Einstein, "Kosmologische Betrachtungen zur allgemeinen Relativitaetstheorie" (Cosmological Considerations in the General Theory of Relativity)", Sitzungsberichte der Königlich Preussischen Akademie der Wissenschaften Berlin, 1917; 142-152

[15] W M Saslow, “A physical interpretation of the Planck length", European Journal of Physics, 1998; 19: 313

[16]Eaton E Lattman, "The Planck length as the dimension of a transient black hole", European Journal of Physics, 2009; 30: L41-L42 
[17] Cornelis P. Dullemond, "Introduction to Cosmology", Lecture Notes, Heidelberg University, Center for Astronomy (ZAH), Institute for Theoretical Astrophysics (ITA), 2020 (www.ita.uni-heidelberg.de/ dullemond/lectures/cosmology_2011/Chapter_9.pdf)

[18] Steven Weinberg, Gravitation and Cosmology: Principles and Applications of the General Theory of relativity, (Wiley India, New Delhi), 2017; p 165

[19] Pavel Voracek, "Relativistic gravitational potential and its relation to mass-energy", Astrophysics and Space Science, 1979; 65:397-413

[20]Planck Collaboration, "Planck 2015 results XIII. Cosmological parameters", Astronomy \& Astrophysics, 2016; A13: 1-63 